\title{
Uso de sustancias estimulantes y comportamiento de juego en adolescentes. Juego y uso de estimulantes
}

\section{Stimulant substance use and gambling behaviour in adolescents. Gambling and stimulant use}

\author{
Alessandra Buja*, Claudia Mortali**, Luisa Mastrobattista**, Elisa De Battisti*, \\ Adele Minutillo**, Simona Pichini**, Giulia Grotto*, Bruno Genetti***, Paolo Vian***, \\ Alessandra Andreotti***, Vincenzo Baldo*, Roberta Pacifici**. \\ * Departamento de ciencias cardiológicas, torácicas, vasculares y salud pública. Universidad de Padua, Padua. Italia. \\ ** Centro Nacional de Dependencia y Dopaje. Instituto Superior de Sanidad, Roma. Italia. \\ *** Centro Explora para investigación y análisis estadísticos, Vigodarzere (PD). Italia.
}

\section{Resumen}

El juego es un importante problema de salud pública ampliamente reconocido. A pesar del creciente uso de sustancias estimulantes entre los adolescentes, todavía son escasos los estudios centrados en verificar la existencia de una asociación entre el uso de estimulantes y los comportamientos relacionados con el juego en adolescentes. Por tanto, este estudio tuvo como objetivo investigar la asociación entre los hábitos relacionados con el juego y el consumo de sustancias estimulantes como el café, las bebidas energizantes y las nuevas sustancias psicoactivas en una muestra de adolescentes italianos. En 2017 se realizó una encuesta en una muestra representativa de jóvenes italianos de 14 a 17 años, constituida por 15833 estudiantes provenientes de 201 escuelas de educación secundaria. Se realizó un análisis de regresión logística para evaluar la asociación entre juego de riesgo/juego problemático (R1) y factores predictivos independientes: el modelo incluyó variables independientes (café, bebida energética y consumo de nuevas sustancias psicoactivas) y otras covariables demográficas, del entorno social y de conductas de riesgo. También se realizó un análisis de sensibilidad para examinar una segunda variable dependiente con respecto a cualquier experiencia de conductas relacionadas con el juego (R2). Los adolescentes clasificados como jugadores de riesgo o jugadores con problemas tenían una probabilidad significativamente mayor de consumir bebidas energizantes que los no jugadores o los jugadores sin riesgo. Se observó un patrón similar en el consumo de nuevas sustancias psicoactivas. No se evidenció ninguna asociación significativa con el consumo de café. El análisis de sensibilidad mostró que, en comparación con los no jugadores, el grupo de jugadores tenía mayores probabilidades de consumo frecuente de café, bebidas energéticas y/o nuevas sustancias psicoactivas. La evaluación del juego y el uso de sustancias estimulantes puede proporcionar información importante. Por consiguiente, podría ser necesario tomar medidas para reducir el uso de sustancias estimulantes como parte de los esfuerzos dirigidos a lidiar con los hábitos de juego poco saludables. Palabas clave: Juego; Adolescentes; Abuso de sustancias; Nuevas sustancias psicoactivas.

\begin{abstract}
Gambling is widely recognized as an important public health problem. Despite the rising use of stimulant substances among adolescents, there are still very few studies focusing on whether adolescents' use of stimulants is associated with their gambling behaviour. Therefore, the aim of this study was to investigate the association between gambling habits and consumption of stimulants such as coffee, energy drinks, and new psychoactive substances in a sample of Italian adolescents. A survey was conducted in 2017 with a representative sample of Italians between the ages of 14-17 years, comprising 15,833 students attending 201 secondary schools. Logistic regression analyses were run to assess the association between at-risk/problem gambling (O1) and independent predictors: the model included independent variables (coffee, energy drinks and new psychoactive substance consumption) and covariates (demographic variables, social environment variables and risk-taking behaviour variables). A sensitivity analysis was also conducted to examine a second dependent variable regarding any experience of gambling behaviour (O2). Adolescents who were at-risk gamblers or problem gamblers were significantly more likely to consume energy drinks than non-gamblers or not-at-risk gamblers. A similar pattern was seen for consumption of new psychoactive substances. No significant association emerged with coffee consumption. The sensitivity analysis showed that, compared with non gamblers, the group of gamblers had higher odds for frequent coffee consumption, as well as for consumption of energy drinks and/or new psychoactive substances. Screening for gambling and stimulant use may provide important information, as it may be necessary to take action to reduce stimulant substance use as part of efforts to deal with unhealthy gambling habits.

Key Words: Gambling; Adolescents; Substance abuse; New psychoactive substances.
\end{abstract}




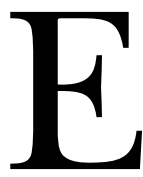

s ampliamente conocido que el juego es un problema importante de salud pública asociado con costes personales y sociales, altas tasas de comorbilidad psiquiátrica, una mala salud física y altas tasas de suicidio (Nautiyal, Okuda, Hen y Blanco, 2017). Existe una asociación entre la exposición temprana al juego durante la adolescencia y problemas más severos en relación al juego más tarde en la vida (Burge, Pietrzark y Petry, 2006). Estudios de prevalencia a gran escala también han confirmado altas tasas de prevalencia de juego y juego problemático entre los jóvenes. Un estudio reciente halló un rango amplio $(35,7-79,1 \%)$ de prevalencia de adolescentes que han jugado en el último año, mientras que se estima que entre $0,2-12,3 \%$ de los adolescentes a nivel mundial tienen juego problemático (Calado, Alexandre y Griffiths, 2017).

Encuestas y revisiones sobre la conducta de juego entre los adolescentes han hallado, de manera consistente, que los jugadores adolescentes tienen una toma de decisiones más impulsiva y rasgos de personalidad de búsqueda de sensaciones (Blinn-Pike, Worthy y Jonkman, 2010; Dowling et al., 2017; Nower, Derevensky y Gupta, 2004). También hay evidencia que sugiere que es más probable que los adolescentes con mayores niveles de ansiedad — como estado tanto como rasgo- se conviertan en jugadores patológicos (Floros, 2018). Las sustancias estimulantes aumentan los niveles de energía y de concentración, pero también pueden afectar los rasgos conductuales y aumentar el temor, la ansiedad, la irritabilidad y la inquietud (Ste-Marie, Gupta y Derevensky, 2006). Algunos estudios, aunque no todos, sobre adolescentes y jóvenes adultos encontraron, en general, que los refrescos con cafeína tienen una asociación positiva con la toma de riesgos, la impulsividad y la búsqueda de sensaciones (Arria et al., 2011; Grant y Chamberlain, 2018; Jones y Lejuez, 2005; Kponee, Siegel y Jernigal, 2014; Temple, Ziegler, Graczyk y Crandall, 2017). En particular, Temple et al. hallaron que la dosis de cafeína era una variable dependiente que afectaba la toma de decisiones y de riesgos. En cambio, en otros estudios pareció ser que la cafeína no alteró la inhibición conductual (medida con una tarea de semáforo (Tieges, Snel, Kok y Richard, 2009)) ni la toma de decisiones (Killgore, Grugle y Balkin, 2009). La publicidad de las bebidas energizantes que contienen cafeína además de otros estimulantes, como guaraná, ephedra, yohimbina, gingko, teofilina y L-carnitina (NFSHSASMAC, 2014) las comercializa como fuentes de mejora de los niveles energéticos, el rendimiento deportivo y la concentración. El mercado y la popularidad de estos productos ha aumentado rápidamente entre los adolescentes: un estudio halló que el 31\% de adolescentes entre 12-17 años informó consumir con regularidad bebidas energizantes (Al-Shaar, Vercammen, Lu, Richardson, Tamez y Mattei, 2017; Seifert, Schaechter, Hershorin y Lipshultz, 2011). Solo pocos estudios han informado que el consumo de bebidas energizantes tuvo una asociación positiva con el juego, en varones adolescentes (Gori et al., 2015) además de en la adolescencia temprana (Gallimberti et al., 2016).

Unas nuevas sustancias psicoactivas (NSP) han tenido un rápido surgimiento en el mercado de los estimulantes en los últimos años (UNODC, 2013). Aunque la mayoría de las NSP son sustancias químicas sintéticas, muchas son de origen vegetal (Feng, Battulga, Han y Chung, 2017).

En décadas recientes, estas sustancias se han introducido en los mercados a través de diversos modos de distribución, incluyendo el Internet, «smart shops» que venden parafernalia para el consumo de drogas o traficantes callejeros como alternativas legales a sustancias ilícitas.

Supone un reto estimar la prevalencia de las NSP debido a las inconsistencias en su metodología y definición, por lo que es difícil comparar las estimaciones nacionales. Un estudio europeo reciente que recogió datos comparables sobre el uso de sustancias entre estudiantes de 15-16 años en 48 países europeos encontró que la media de experiencias con las NSP a lo largo de la vida fue del 4\%, con la tasa en Italia de $6 \%$ (ESPAD, 2016). Se había demostrado anteriormente los efectos paralelos de la dopamina sobre el juego, y de los psicoestimulantes sobre diversos ámbitos (refuerzo de recompensa, «priming» motivacional, y procesamiento de información subjetivo, experiencial y cognitivo) (Zack y Poulos, 2009), pero hasta la fecha ningún estudio ha analizado la asociación entre el consumo de dichas drogas y el juego.

Por tanto, este estudio tuvo como objetivo investigar el vínculo entre los hábitos de juego y el consumo de sustancias estimulantes, como café, bebidas energizantes y las nuevas sustancias psicoactivas en una muestra de adolescentes italianos.

\section{Métodos}

La población muestral fue extraída del proyecto «Gambling in Italy», una encuesta sobre el juego realizada con estudiantes en 2017 por el Instituto Superior de Sanidad. Para los fines de este estudio, presentamos abajo una descripción breve de la encuesta.

\section{Muestra}

La muestra se compuso de la población estudiantil italiana entre 14-17 años, teniendo en cuenta la distribución geográfica nacional de la población para interceptar áreas metropolitanas, urbanas y suburbanas. El método de muestreo siguió un modelo trifásico de PPT (Probabilidad Proporcional al Tamaño) donde las unidades de la primera fase se representaron por ciudades, las unidades de la segunda fase se representaron por escuelas, y las unidades de la tercera fase se representaron por clases. El diseño de muestreo incluyó la estratificación de las unidades primera, segunda y tercera; en cada estrato: las unidades de la primera fase (ciudad) se seleccionaron con probabilidades proporcionales al número de clases superiores de escuelas secundarias en el territorio 
de las ciudades; las unidades de la segunda fase (escuela) se seleccionaron con probabilidades proporcionales al número de clases en las escuelas de la muestra; y las unidades de la tercera fase (clase) se seleccionaron en las mismas cantidades para cada escuela en el estrato al cual pertenecían. Todos los estudiantes que asistían a las clases incluidas en la muestra fueron incluidos. Este método de muestreo permitió mantener constantes la probabilidad de cada clase y cada estudiante en la población objetivo seleccionada.

La encuesta se administró mediante el método de la auto entrevista asistida por computadora (CASI) que permitió a los estudiantes completar el cuestionario en línea mediante una identificación de acceso anónima, única y no replicable. Los estudiantes accedieron al cuestionario a través del enlace facilitado por los técnicos en las aulas de informática de las escuelas.

Participaron en la encuesta 201 escuelas (187 públicas, 14 privadas) con 859 clases como muestra, sumando una población estudiantil de 18 042. Los estudiantes de las escuelas que acordaron participar en la encuesta completaron 17610 encuestas. Se rechazaron seis cuestionarios porque los contestaron estudiantes no residentes en Italia (paso 1); se rechazaron 267 por estar incompletos (paso 2); se rechazaron otros 1504 porque los contestaron estudiantes fuera del grupo de edad abarcado por la encuesta (i.e., menores de 14 años o mayores de 17 años) (paso 3) y se rechazaron 231 porque contenían respuestas consideradas poco plausibles, i.e., se identificaron las respuestas no fiables o irrelevantes mediante un análisis del modelo de Rasch (paso 4). Por tanto, 15602 cuestionarios (el 88,6\% del total) fueron admitidos al estudio.

\section{Variables}

Usamos la escala SOGS-RA (Poulin, 2002; Winters, Stinchfield y Fulkerson, 1993) para examinar la conducta de juego de los encuestados. Esta herramienta validada contiene 12 ítems y puntuaciones entre 0 y 12 . Mide varios aspectos, como pérdida de control sobre el juego, acciones para recuperar las pérdidas económicas, interferencia con la familia, los estudios y las relaciones sociales, sentimientos de culpabilidad respecto del dinero gastado y las consecuencias del juego. Para ser definidos como «jugadores», los encuestados tuvieron que informar haber participado en una actividad de juego al menos una vez en el último año. Por tanto, la escala SOGS-RA identifica tres tipos de jugadores: sin problema (puntuación SOGS-RA = 0-1); de riesgo (puntuación SOGS-RA = 2-3); y problemático (puntuación SOGS-RA = > 4). Los estudiantes que informaron no haber tenido experiencia alguna con el juego en el último año se definieron como «no jugadores».

Las variables independientes analizadas tuvieron en cuenta el consumo de bebidas energizantes (BE), nuevas sustancias psicoactivas (NSP) y café (C). Se clasificó cada variable según el uso autoinformado de los encuestados en una de seis categorías: 1. «nunca»; 2. «rara vez, en ocasiones especiales»; 3. «algunos días entre semana (lunes a viernes)»; 4. «solo durante fines de semana»; 5. «algunos días entre semana (lunes a viernes) y durante fines de semana»; y 6. «todos los días de la semana».

Las variables demográficas consideradas fueron: edad, sexo, nacionalidad (italiana, país de la UE, otros países). En cuanto al entorno social de los encuestados, respondieron a preguntas sobre el nivel económico de su familia (por encima, igual, o por debajo del nivel de las familias de sus amistades); cantidad semanal de dinero para gastos (€0-20, $€ 21-50, €_{i} 50$ ); perfil en redes sociales (sí, no); rendimiento académico (pobre, medio, bueno o muy bueno). El cuestionario también incluyó preguntas acerca de otros usos de sustancias relacionadas con el riesgo: tabaquismo (nunca haber fumado, ex-fumador, fumador ocasional, fumador a diario) e ingesta de alcohol como cerveza/vino/cócteles/ licores (nunca, de vez en cuando pero menos de una vez el mes, con frecuencia entre cada mes y todos los días).

\section{Análisis estadístico}

El análisis no usó un enfoque de estadística complejo. Dado el gran tamaño muestral, adoptamos el método de Bernoulli de muestro aleatorio simple. Se hizo un análisis bivariable para cada una de las variables descritas arriba y estado de juego. Usamos una serie de tests de chi cuadrado de Pearson para destacar cualquier asociación entre juego y otras variables.

Hicimos análisis de regresión logística parar valorar la asociación entre resultado (estado de juego Resultado 1 $(\mathrm{R} 1)$ = no jugadores y jugadores sin riesgo versus jugadores de riesgo o jugadores problemáticos, como definimos anteriormente según las puntuaciones en el SOGS-RA) y predictores. El modelo contuvo variables independientes (café, bebidas energizantes y nuevas sustancias psicoactivas) en la regresión como variables ficticias para consumo (nunca; rara vez, en ocasiones especiales; demás modalidades) y las covariables (variables demográficas, del entorno social y de conductas de riesgo).

También realizamos un análisis de sensibilidad al valorar una segunda variable dependiente (estado de juego Resultado 2 (R2) = no jugadores versus jugadores sin riesgo, de riesgo o problemáticos, como definimos anteriormente según las puntuaciones en el SOGS-RA), e incluimos los mismos predictores independientes y las covariables en el modelo subsecuente.

\section{Cuestiones éticas}

El estudio se hizo conforme a la Declaración de Helsinki y la ley italiana n. ${ }^{\circ}$ 196/2003 sobre la protección de datos personales. Los datos se recogieron de forma anónima y los análisis se realizaron sobre los datos agregados, sin la posibilidad de poder identificar a los individuos. Primero, el director de la escuela tuvo que dar su consentimiento 
para que los estudiantes pudiesen participar. Después, los padres firmaron un formulario de consentimiento para que los menores pudieran participar en la encuesta.

\section{Resultados}

Analizamos 15602 cuestionarios. Tabla 1 muestra las características de la muestra.

La prevalencia de los no jugadores fue $70,8 \%$ (IC 95\% 69,8-71,8), mientras que el 22,7\% (IC 95\% 21,8-23,6) de los estudiantes reportaron ser jugadores sin riesgo, el 3,5\% (IC 95\% 3,1-3,9) fueron jugadores de riesgo, y el 3,0\% (IC 95\% 2,7-3,4) fueron jugadores problemáticos.

Tabla 2 muestra los resultados del análisis bivariable de las conductas de juego y las distintas covariables. Entre los jugadores de riesgo/problemáticos, el $86,9 \%$ eran varones, el $35,8 \%$ consumían bebidas energizantes con frecuencia (mientras que esto era el caso para solo el 14,8\% de los no jugadores y los jugadores sin riesgo; valor $p<$ ,001). También surgió una diferencia estadísticamente significativa de $1,5 \%$ de los no jugadores y los jugadores sin riesgo, comparado con el $8,5 \%$ de los jugadores de riesgo y jugadores problemáticos, que usaban con frecuencia las nuevas sustancias psicoactivas; valor $p<, 001$. Fue más baja la diferencia entre ambos grupos de jugadores en cuanto al consumo frecuente de café $(56,6 \%$ vs $67,6 \%)$, pero todavía estadísticamente significativa (valor $p<, 001$ ).

Tabla 3 muestra los resultados del análisis de regresión logística para cada resultado. En comparación con los no jugadores o los jugadores sin riesgo (R1), era significativamente más probable que los jugadores de riesgo o problemáticos consumieran bebidas energizantes (rara vez: RM 1,28, IC $95 \%$ 1,08-1,52; con frecuencia: RM 1,95, IC $95 \%$ 1,62-2,34). Emergieron patrones similares entre los dos grupos para el consumo de las nuevas sustancias psicoactivas (rara vez: RM 1,37, IC $95 \%$ 0,99-1,89; con frecuencia: RM: 2,96, IC 95 \% 2,21-3,95). No hubo una asociación significativa para el consumo de café.

El análisis de sensibilidad mostró que, en comparación con el grupo de no jugadores, el grupo de jugadores (R2) también tuvo una asociación positiva con el consumo frecuente de café (RM 1,20, IC 95\% 1,09-1,33), además de con el consumo de bebidas energizantes rara vez y con frecuencia (RM 1,44, IC 95\% 1,32-1,58 y RM 1,75, IC $95 \%$ $1,57-1,95)$ y consumo frecuente de las nuevas sustancias psicoactivas (RM 2,02, IC 95\% 1,58-2,60).

\section{Discusión}

Nuestro estudio muestra que los adolescentes con experiencia en el juego de riesgo y problemático tienen mayor probabilidad de ser consumidores de bebidas energizantes y de las nuevas sustancias psicoactivas, tras ajustar por factores sociodemográficos y el consumo de otras sustancias (tabaquismo y consumo de alcohol). El consumo frecuen-
Tabla 1. Características de la muestra de adolescentes.

\begin{tabular}{|c|c|c|}
\hline & \multicolumn{2}{|c|}{ Total N = 15602} \\
\hline & $\mathbf{N}$ & $\%$ \\
\hline \multicolumn{3}{|l|}{ Sexo } \\
\hline Hombre & 7662 & 49,1 \\
\hline Mujer & 7940 & 50,9 \\
\hline \multicolumn{3}{|l|}{ Edad } \\
\hline 14 años & 3690 & 23,7 \\
\hline 15 años & 3932 & 25,2 \\
\hline 16 años & 4008 & 25,7 \\
\hline 17 años & 3972 & 25,5 \\
\hline \multicolumn{3}{|l|}{ Nacionalidad } \\
\hline Italiana & 14793 & 94,8 \\
\hline Países de la UE & 217 & 1,4 \\
\hline Otros países & 592 & 3,8 \\
\hline \multicolumn{3}{|l|}{ Nivel económico de la familia } \\
\hline Desconocido & 1359 & 8,7 \\
\hline Por encima de las familias de las amistades & 1552 & 9,9 \\
\hline Igual que las familias de las amistades & 11502 & 73,7 \\
\hline Por debajo de las familias de las amistades & 1189 & 7,6 \\
\hline \multicolumn{3}{|l|}{ Dinero semanal para gastos } \\
\hline$€ 0-20$ & 11788 & 75,6 \\
\hline$€ 21-50$ & 3003 & 19,2 \\
\hline$>€ 50$ & 811 & 5,2 \\
\hline \multicolumn{3}{|l|}{ Rendimiento académico } \\
\hline Pobre & 403 & 2,6 \\
\hline Medio & 11066 & 70,9 \\
\hline Bueno o muy bueno & 4133 & 26,5 \\
\hline \multicolumn{3}{|l|}{ Perfil en «redes sociales» } \\
\hline No & 446 & 2,9 \\
\hline Sí & 15156 & 97,1 \\
\hline \multicolumn{3}{|l|}{ Tabaquismo } \\
\hline Nunca haber fumado & 8103 & 51,9 \\
\hline Ex-fumador & 326 & 2,1 \\
\hline Fumador ocasional & 5356 & 34,3 \\
\hline Fumador a diario & 1817 & 11,6 \\
\hline \multicolumn{3}{|l|}{ Conducta de ingesta de alcohol } \\
\hline Nunca & 3999 & 25,6 \\
\hline De vez en cuando pero menos de una vez el mes & 4523 & 29,0 \\
\hline Con frecuencia entre cada mes y todos los días & 7080 & 45,4 \\
\hline \multicolumn{3}{|l|}{ Consumo de bebidas energizantes } \\
\hline Nunca & 7926 & 50,8 \\
\hline Rara vez & 5155 & 33,0 \\
\hline Con frecuencia & 2521 & 16,2 \\
\hline \multicolumn{3}{|l|}{ Consumo de las nuevas sustancias psicoactivas } \\
\hline Nunca & 14952 & 95,8 \\
\hline Rara vez & 339 & 2,2 \\
\hline Con frecuencia & 311 & 2,0 \\
\hline \multicolumn{3}{|l|}{ Consumo de café } \\
\hline Nunca & 3586 & 23,0 \\
\hline Rara vez & 3071 & 19,7 \\
\hline Con frecuencia & 8945 & 57,3 \\
\hline
\end{tabular}


Alessandra Buja, Claudia Mortali, Luisa Mastrobattista, Elisa De Battisti, Adele Minutillo, Simona Pichini, Giulia Grotto, Bruno Genetti, Paolo Vian, Alessandra Andreotti, Vincenzo Baldo, Roberta Pacifici

Tabla 2. Análisis bivariado.

\begin{tabular}{|c|c|c|c|}
\hline & $\begin{array}{l}\text { No jugador/Jugador sin riesgo } \\
(\mathrm{N}=14590)\end{array}$ & $\begin{array}{l}\text { Jugador de riesgo/Jugador problemático } \\
\qquad(\mathrm{N}=1012)\end{array}$ & Valor $\mathrm{p}$ \\
\hline \multicolumn{4}{|l|}{ Sexo } \\
\hline Hombre & $46,5 \%(6783)$ & $86,9 \%(879)$ & \multirow{2}{*}{$<, 001$} \\
\hline Mujer & $53,5 \%(7807)$ & $13,1 \%(133)$ & \\
\hline \multicolumn{4}{|l|}{ Edad } \\
\hline 14 años & $24,4 \%(3558)$ & $13,0 \%(132)$ & \multirow{4}{*}{$<, 001$} \\
\hline 15 años & $25,3 \%(3694)$ & $23,5 \%(238)$ & \\
\hline 16 años & $25,6 \%(3729)$ & $27,6 \%(279)$ & \\
\hline 17 años & $24,7 \%(3609)$ & $35,9 \%(363)$ & \\
\hline \multicolumn{4}{|l|}{ Nacionalidad } \\
\hline Italiana & $94,8 \%(13829)$ & $95,3 \%(964)$ & \multirow{3}{*}{,755 } \\
\hline Países de la UE & $1,4 \%(203)$ & $1,4 \%(14)$ & \\
\hline Otros países & $3,8 \%(558)$ & $3,4 \%(34)$ & \\
\hline \multicolumn{4}{|l|}{ Nivel económico de la familia } \\
\hline Desconocido & $8,7 \%(1267)$ & $9,1 \%(92)$ & \multirow{4}{*}{$<, 001$} \\
\hline Por encima de las familias de las amistades & $9,6 \%(1403)$ & $14,7 \%(149)$ & \\
\hline Igual que las familias de las amistades & $74,1 \%(10816)$ & $67,8 \%(686)$ & \\
\hline Por debajo de las familias de las amistades & $7,6 \%(1104)$ & $8,4 \%(85)$ & \\
\hline \multicolumn{4}{|l|}{ Dinero semanal para gastos } \\
\hline$€ 0-20$ & $76,9 \%(11224)$ & $55,7 \%(564)$ & \multirow{3}{*}{$<, 001$} \\
\hline$€ 21-50$ & $18,6 \%(2716)$ & $28,4 \%(287)$ & \\
\hline$>€ 50$ & $4,5 \%(650)$ & $15,9 \%(161)$ & \\
\hline \multicolumn{4}{|l|}{ Rendimiento académico } \\
\hline Pobre & $2,4 \%(348)$ & $5,4 \%(55)$ & \multirow{3}{*}{$<, 001$} \\
\hline Medio & $70,5 \%(10284)$ & $77,3 \%(782)$ & \\
\hline Bueno o muy bueno & $27,1 \%(3958)$ & $17,3 \%(175)$ & \\
\hline \multicolumn{4}{|l|}{ Perfil en «redes sociales» } \\
\hline No & $2,9 \%(423)$ & $2,3 \%(23)$ & \multirow{2}{*}{, 247} \\
\hline Sí & $97,1 \%(14167)$ & $97,7 \%(989)$ & \\
\hline \multicolumn{4}{|l|}{ Tabaquismo } \\
\hline Nunca haber fumado & $53,5 \%(7808)$ & $29,2 \%(295)$ & \multirow{4}{*}{$<, 001$} \\
\hline Ex-fumador & $2,0 \%(287)$ & $3,9 \%(39)$ & \\
\hline Fumador ocasional & $33,9 \%(4942)$ & $40,9 \%(414)$ & \\
\hline Fumador a diario & $10,6 \%(1553)$ & $26,1 \%(264)$ & \\
\hline \multicolumn{4}{|l|}{ Conducta de ingesta de alcohol } \\
\hline Nunca & $26,9 \%(3924)$ & $7,4 \%(75)$ & \multirow{3}{*}{$<, 001$} \\
\hline De vez en cuando pero menos de una vez el mes & $29,9 \%(4359)$ & $16,2 \%(164)$ & \\
\hline Con frecuencia entre cada mes y todos los días & $43,2 \%(6307)$ & $76,4 \%(773)$ & \\
\hline \multicolumn{4}{|l|}{ Consumo de bebidas energizantes } \\
\hline Nunca & $52,5 \%(7655)$ & $26,8 \%(271)$ & \multirow{3}{*}{$<, 001$} \\
\hline Rara vez & $32,7 \%(4776)$ & $37,5 \%(379)$ & \\
\hline Con frecuencia & $14,8 \%(2159)$ & $35,8 \%(362)$ & \\
\hline \multicolumn{4}{|l|}{ Consumo de las nuevas sustancias psicoactivas } \\
\hline Nunca & $96,5 \%(14079)$ & $86,3 \%(873)$ & \multirow{3}{*}{$<, 001$} \\
\hline Rara vez & $2,0 \%(286)$ & $5,2 \%(53)$ & \\
\hline Con frecuencia & $1,5 \%(225)$ & $8,5 \%(86)$ & \\
\hline Consumo de café & & & \\
\hline Nunca & $23,5 \%(3424)$ & $16,0 \%(162)$ & \\
\hline Rara vez & $19,9 \%(2905)$ & $16,4 \%(166)$ & $<, 001$ \\
\hline Con frecuencia & $56,6 \%(8261)$ & $67,6 \%(684)$ & \\
\hline
\end{tabular}


Tabla 3. Análisis de regresión logística binaria.

\begin{tabular}{|c|c|c|c|c|}
\hline & \multirow[b]{2}{*}{$\mathrm{OR}^{*}$} & \multicolumn{2}{|c|}{ IC $95 \%$} & \multirow[b]{2}{*}{ Valor $\mathrm{p}$} \\
\hline & & $\begin{array}{l}\text { Límite } \\
\text { inferior }\end{array}$ & $\begin{array}{l}\text { Límite } \\
\text { superior }\end{array}$ & \\
\hline \multicolumn{5}{|c|}{ R1 = jugador de riesgo/jugador problemático } \\
\hline \multicolumn{5}{|c|}{ Bebidas energizantes } \\
\hline Rara vez & 1,28 & 1,08 & 1,52 &, 004 \\
\hline Con frecuencia & 1,95 & 1,62 & 2,34 & $<, 001$ \\
\hline \multicolumn{5}{|c|}{ Nuevas sustancias psicoactivas } \\
\hline Rara vez & 1,37 & ,99 & 1,89 &, 057 \\
\hline Con frecuencia & 2,96 & 2,21 & 3,95 & $<, 001$ \\
\hline \multicolumn{5}{|l|}{ Café } \\
\hline Rara vez & 1,10 & ,87 & 1,38 & ,446 \\
\hline Con frecuencia & 1,03 &, 85 & 1,25 & ,734 \\
\hline \multicolumn{5}{|c|}{ R2 = cualquier conducta de juego } \\
\hline \multicolumn{5}{|c|}{ Bebidas energizantes } \\
\hline Rara vez & 1,44 & 1,32 & 1,58 & $<, 001$ \\
\hline Con frecuencia & 1,75 & 1,57 & 1,95 & $<, 001$ \\
\hline \multicolumn{5}{|c|}{ Nuevas sustancias psicoactivas } \\
\hline Rara vez & 1,14 & ,90 & 1,44 &, 274 \\
\hline Con frecuencia & 2,02 & 1,58 & 2,60 & $<, 001$ \\
\hline \multicolumn{5}{|l|}{ Café } \\
\hline Rara vez & 1,09 & ,97 & 1,24 & 157, \\
\hline Con frecuencia & 1,20 & 1,09 & 1,33 & $<, 001$ \\
\hline
\end{tabular}

Nota. $\mathrm{OR}=$ Odds Ratio; IC = Intervalo de Confianza

*Los análisis de regresión se ajustaron por las variables: sexo, edad, nacionalidad, nivel económico de la familia, dinero semanal para gastos, rendimiento académico, tabaquismo y consumo de alcohol.

te de café también está asociado con cualquier experiencia de juego en adolescentes.

Nuestros datos muestran que casi una tercera parte de los adolescentes participa en el juego. A pesar de las restricciones legales respecto de edad, los menores y los adolescentes pueden fácilmente acceder a varias oportunidades de juego ilícito, y muchos lo hacen (Malgorzata Carran, 2013). Estos hallazgos merecen atención porque los estudios con poblaciones adultas han mostrado que los jugadores patológicos adultos muchas veces empezaron su actividad de juego a una edad relativamente temprana, y que cuanto antes se inicia en el juego, más probabilidad de que sean jugadores problemáticos (Burge et al., 2006).

También encontramos una asociación entre el consumo de café y cualquier experiencia de juego en adolescentes. De manera similar, un estudio con estudiantes universitarios (Temple et al., 2017) señaló una relación especialmente fuerte entre la ingesta de cafeína, edad más joven de las primeras experiencias de juego y ciertos tipos de impulsividad entre los jugadores. En otro estudio con jóvenes adultos, hubo una correlación positiva significativa entre la ingesta diaria media de cafeína y las puntuaciones en un cuestionario sobre la toma de riesgos (Ste-Marie, Gupta y Derevensky, 2006).
También hallamos una asociación entre el consumo de bebidas energizantes y conductas de juego de riesgo y problemáticas en la adolescencia. Las bebidas energizantes contienen entre 75-158 mg de cafeína por lata, y estas bebidas tienen efectos estimulantes en el sistema nervioso central (SNC). La motivación por tomarlas es la expectativa de que mejoran el rendimiento físico y mental del consumidor. De hecho, mediante el antagonismo de la adenosina A1 y los receptores A2A, la cafeína combate los efectos inhibitorios de la adenosina en la dopamina, y así aumenta la psicoactividad de los sistemas dopaminérgicos D1 y D2, con efectos en el estado de ánimo, la función ejecutiva, la atribución de saliencia, la cognición y la regulación conductual (Ishak, Ugochukwu, Bagot, Khalili y Zaky, 2012). Parece ser que las conductas de toma de riesgos se manifiestan en la adolescencia debido a la mayor búsqueda de sensaciones relacionada con los cambios en la actividad dopaminérgica entre la niñez y la adolescencia (Arenas et al., 2016). Ya se mostró anteriormente el papel paralelo y dominante de la dopamina en relación a la fisiopatología del juego, y se describió como psicoestimulante-mimético (Zack y Poulos, 2009). Una revisión reciente del consumo de bebidas energizantes aportó evidencia de que los consumidores eran tomadores de alto riesgo y más propensos a exhibir conductas de un estilo de vida caracterizadas por la desinhibición y la falta de moderación, incluyendo tabaquismo, consumo de alcohol y cafeína, además de juego (Verster et al., 2018).

Por último, nuestro estudio muestra que hay una asociación entre el juego de riesgo y problemático en la adolescencia y el consumo de las nuevas sustancias psicoactivas. Hasta la fecha, pocos estudios se han hecho sobre el uso de las nuevas sustancias psicoactivas por los adolescentes. Un estudio con estudiantes universitarios mostró que los que habían usado algún tipo de sustancia estimulante en los últimos 3 meses tenían probabilidades un $74 \%$ más altas de haber tenido conductas de juego problemático en los últimos 6 meses (Geisner et al., 2016). Otro estudio con estudiantes de secundaria entre 12-19 años en los EE. UU. también encontró una asociación entre el uso de sustancias estimulantes y probabilidades más altas de mayor frecuencia de juego y juego problemático, en ambos sexos. El modelo psicoestimulante-mimético predice que las drogas estimulantes hacen de «priming» cruzado con la motivación para jugar (Zack y Poulos, 2009). De manera coherente, Zack (2004) et al. aportaron evidencia experimental de una activación neuroquímica similar a la inducida por las drogas psicoestimulantes como una característica importante de la adicción al juego. Varios informes sugieren que el juego puede inducir efectos muy parecidos a los de una droga psicoestimulante, y que los perfiles de un episodio de juego y el uso de drogas psicoestimulantes también son parecidos: ambos conllevan una conducta marcada de perseveración (Dickerson, Hinchy y Fabre, 1987). Dicha evidencia implica que las drogas psicoestimulantes y la participación en el juego provocan muchos de los mismos efectos. 
Si es así, la dosis de una droga psicoestimulante puede hacer de «priming» de la motivación para jugar, de la misma manera que una «dosis» de juego (Zack y Poulos, 2004). Los estudios de neuroimagen también indican que la expectativa de recibir dinero induce unos patrones selectivos de activación en las vías dopamínicas cerebrales (Knutson, Fong, Adams, Varner y Hommer, 2001). Estas vías también tienen una importante implicación en los efectos de refuerzo de las drogas psicoestimulantes (Mackey \& van der Kooy, 1985; Spyraki, Fibiger y Phillips, 1982; Yokel y Wise, 1978). Otro asunto a tener en cuenta es los rasgos de personalidad: sentimientos de alienación, ansiedad, baja autoestima y actitudes hacia la desviación, independencia e impulsividad podrían aumentar el riesgo tanto de juego problemático como del uso de drogas estimulantes (Brezing, Derevensky y Potenza, 2010; Jessor, 1987; Romer, 2003; Secades-Villa, Garcia-Rodriguez, Jin, Wang y Blanco, 2015).

Este estudio tiene varias limitaciones, principalmente en relación al hecho de que obtuvimos nuestros datos de una muestra de adolescentes escolarizados. Esto implica que cualquier alumno que abandonó la escuela con 16 años (al finalizar los estudios obligatorios en Italia) quedaron excluidos, por lo que nuestra muestra solo es representativa de estudiantes italianos. Una segunda limitación inherente a nuestros hallazgos es que se basan en autoinformes y las respuestas de los encuestados pueden estar sesgadas al reportar sus conductas de toma de riesgos por encima o por debajo de la realidad. Garantizamos la anonimidad y la confidencialidad de los encuestados con el fin de mitigar este posible sesgo. Tercero, el diseño transversal de este estudio limitó nuestra capacidad para hacer inferencias causales, en particular respecto de la direccionalidad de la asociación entre el uso de sustancias estimulantes y el juego, que puede ser bidireccional.

\section{Conclusión}

El consumo entre adolescentes de sustancias estimulantes —como las nuevas sustancias psicoactivas y las bebidas energizantes - está asociado con juego de riesgo/ problemático. Es sabido que la adolescencia coincide con un impulso natural de buscar sensaciones y tomar riesgos, y el juego y/o el uso de estimulantes puede exacerbar esta tendencia natural con implicaciones psicológicas, sociales y económicas potencialmente negativas. Dada la asociación significativa identificada entre el uso de sustancias estimulantes y actividades de juego entre adolescentes, sería importante organizar esquemas educativos que mejoren la concienciación de las personas acerca de los solapamientos entre muchos tipos de conductas de riesgo.

Asesores en salud mental, trabajadores sociales y psicólogos que trabajan con estudiantes de secundaria y otros adolescentes necesitan tener en cuenta las asociaciones significativas entre las conductas de riesgo. Cribar a los adolescentes tanto para actividad de juego como para el uso de estimulan- tes puede aportar información útil sobre las acciones a implementar para reducir el consumo de sustancias estimulantes entre jóvenes, también como parte de los esfuerzos para prevenir y gestionar problemas relacionados con el juego.

\section{Reconocimientos}

No aplicable.

\section{Conflicto de interés}

Los autores no tienen relación alguna financiera ni de otro tipo relevante a divulgar en relación a este manuscrito.

\section{Referencias}

Al-Shaar, L., Vercammen, K., Lu, C., Richardson, S., Tamez, M. y Mattei, J. (2017). Health Effects and public health concerns of energy drink consumption in the United States: a mini-review. Frontiers in Public Health, 31, 5-225. doi:10.3389/fpubh.2017.00225.

Arenas, M. C., Aguilar, M. A., Montagud-Romero, S., Mateos-García A., Navarro-Francés C. I., Miñarro, J. y Rodríguez-Arias, M. (2016). Influence of the novelty-seeking endophenotype on the rewarding effects of psychostimulant drugs in animal models. Current Neuropharmacology, 14, 87-100.

Arria, A. M., Caldeira, K. M., Kasperski, S. J., Vincent, K. B., Griffiths, R. R. y O’Grady, K. E. (2011). Energy drink consumption and increased risk for alcohol dependence. Alcoholism: Clinical and Experimental Research, 35, 365375. doi:10.1111/j.1530-0277.2010.01352.x.

Blinn-Pike, L., Worthy, S. L. y Jonkman, J. N. (2010). Adolescent gambling: A review of an emerging field of research. The Journal of Adolescent Health. 47, 223-236. doi:10.1016/j.jadohealth.2010.05.003.

Brezing, C., Derevensky, J. L. y Potenza, M. N. (2010). Non-substance-addictive behaviors in youth: pathological gambling and problematic Internet use. Child $\mathcal{E}^{2}$ Adolescent Psychiatric Clinics of North America, 19, 625-641. doi:10.1016/j.chc.2010.03.012.

Burge, A. N., Pietrzak, R. H. y Petry, N. M. (2006). Pre/ Early adolescent onset of gambling and psychosocial problems in treatment-seeking pathological gamblers. Journal of Gambling Studies, 22, 263-274.

Calado, F., Alexandre, J. y Griffiths, M. D. (2017). Prevalence of adolescent problem gambling: A systematic review of recent research. Journal of Gambling Studies, 33, 397-424. doi:10.1007/s10899-016-9627-5.

Dickerson, M., Hinchy, J. y Fabre, J. (1987). Chasing, arousal and sensation seeking in off-course gamblers. British Journal of Addiction, 82, 673-680.

Dowling, N.A., Merkouris, S. S., Greenwood, C. J., Oldenhof, E., Toumbourou, J. W. y Youssef, G. J. (2017). Early risk and protective factors for problem gambling: A systematic review and meta-analysis of longitudinal studies. Clinical Psychology Review, 51, 109-124. doi:10.1016/j. cpr.2016.10.008.

Feng, L. Y, Battulga, A., Han, E. y Chung H. (2017). New psychoactive substances of natural origin: a brief re- 
view. Journal of Food and Drug Analysis, 25, 461-471. doi:10.1016/j.jfda.2017.04.001.

Floros, G. D. (2018). Gambling disorder in adolescents: prevalence, new developments, and treatment challenges. Adolescent Health, Medicine and Therapeutics, 2, 43-51. doi:10.2147/AHMT.S135423.

Gallimberti, L., Buja, A., Chindamo, S., Terraneo, A., Marini, E., Gomez Perez, L. J. y Baldo, V. (2016). Experience with gambling in late childhood and early adolescence: implications for substance experimentation behavior. Journal of Developmental and Behavioral Pediatrics, 37, 148156. doi:10.1097/DBP.0000000000000252.

Geisner, I. M., Huh, D., Cronce, J. M., Lostutter, T. W., Kilmer, J. y Larimer, M. E. (2016). Exploring the relationship between stimulant use and gambling in college students. Journal of Gambling Studies, 32, 1001-1016. doi:10.1007/s10899-015-9586-2.

Gori, M., Potente, R., Pitino, A., Scalese, M., Bastiani, L. y Molinaro, S. (2015). Relationship between gambling severity and attitudes in adolescents: findings from a population-based study. Journal of Gambling Studies, 31, 717-740. doi:10.1007/s10899-014-9481-2.

Grant, J. E. y Chamberlain, S. R. (2018). Caffeine's influence on gambling behavior and other types of impulsivity. Addictive Behaviors, 76, 156-160. doi:10.1016/j.addbeh.2017.08.007.

Ishak, W. W., Ugochukwu, C., Bagot, K., Khalili, D. y Zaky, C. (2012). Energy drinks: psychological effects and impact on well-being and quality of life-a literature review. Innovations in Clinical Neuroscience, 9, 25-34.

Jessor R. (1987). Problem-behavior theory, psychosocial development, and adolescent problem drinking. British Journal of Addiction. 82, 331-342. doi:10.1111/j.1360-0443.1987.tb01490.x.

Jones, H. A. y Lejuez, C. W. (2005). Personality correlates of caffeine dependence: the role of sensation seeking, impulsivity, and risk taking. Experimental and Clinical Psychopharmacology, 13, 259-266.

Killgore, W. D., Grugle, N. L. y Balkin, T. J. (2009) Gambling when sleep deprived: don't bet on stimulants. Chronobiology International, 29, 43-54. doi:10.3109/0742 0528.2011.635230.

Knutson, B., Fong, G. W., Adams, C. M., Varner, J. L. y Hommer, D. (2001). Dissociation of reward anticipation and outcome with event-related fMRI. Neuroreport, 12, 3683-3687.

Kponee, K. Z., Siegel, M. y Jernigan, D. H. (2014). The use of caffeinated alcoholic beverages among underage drinkers: results of a national survey. Addictive Behaviors, 39, 253-258.

Mackey, W. B. y van der Kooy. D. (1985). Neuroleptics block the positive reinforcing effects of amphetamine but not of morphine as measured by place conditioning. Pharmacology Biochemistry and Behavior, 22, 101-105.

Malgorzata Carran, M. (2013). Minors and Gambling Regulation. European Journal of Risk Regulation, 4, 509-520. doi.10.1017/S1867299X00003135.

Nautiyal, K. M., Okuda, M., Hen, R. y Blanco, C. (2017). Gambling disorder: an integrative review of animal and human studies. Annals of the New York Academy of Sciences, 1394, 106-127. doi:10.1111/nyas.13356.

Nower, L., Derevensky, J. L. y Gupta, R. (2004). The relationship of impulsivity, sensation seeking, coping, and substance use in youth gamblers. Psychology of Addictive Behaviors, 18, 49-55.

Poulin, C. (2002). An assessment of the validity and reliability of the SOGS-RA. Journal of Gambling Studies, 18, 67-93.

Romer, D. (2003). Reducing adolescent risk: toward an integrated approach. Sage Publications; Thousand Oaks, CA. Recuperado de https://sk.sagepub.com/books/reducing-adolescent-risk. doi:10.4135/9781452233611.

Secades-Villa, R., Garcia-Rodríguez, O., Jin, C. J., Wang, S. y Blanco, C. (2015). Probability and predictors of the cannabis gateway effect: a national study. International Journal of Drug Policy, 26, 135-142. doi:10.1016/j.drugpo.2014.07.011.

Seifert, S. M., Schaechter, J. L., Hershorin, E. R. y Lipshultz, S. E. (2011). Health effects of energy drinks on children, adolescents, and young adults. Pediatrics, 127, 511-528. doi:10.1542/peds.2009-3592.

Spyraki, C., Fibiger, H. C. y Phillips, A. G. (1982). Dopaminergic substrates of amphetamine-induced place preference conditioning. Brain Research, 253, 185-193.

Ste-Marie, C., Gupta, R. y Derevensky, J. L. (2006). Anxiety and social stress related to adolescent gambling behavior and substance use. Journal of Child $\mathcal{E}^{2}$ Adolescent Substance Abuse, 15, 55-74.

Temple, J. L., Ziegler, A. M., Graczyk, A. M. y Crandall, A. (2017). Effects of acute and chronic caffeine on risk-taking behavior in children and adolescents. Journal of Psychopharmacology, 31, 561-568. doi:10.1177/0269881117691568.

The ESPAD Group. (2016). ESPAD Report 2015. Results from the European School Survey Project on alcohol and other drugs. Luxembourg: Publications Office of the European Union.

Tieges, Z., Snel, J., Kok, A. y Richard, R. K. (2009). Caffeine does not modulate inhibitory control. Brain Cognition, 69, 316-327. doi:10.1016/j.bandc.2008.08.001.

UNODC. (2013). The challenge of new psychoactive substances. A Report from the Global SMART Programme. UNODC, Vienna.

Verster, J. C., Benson, S., Johnson, S. J., Alford, C., Godefroy, S. B. y Scholey, A. (2018). Alcohol mixed with energy drink (AMED): a critical review and meta-analysis. Human Psychopharmacology Clinical and Experimental, 33, 2650. doi:10.1002/hup.2650.

Winters, K. C., Stinchfield, R. D. y Fulkerson, J. (1993). Toward the development of an adolescent gambling problem severity scale. Journal of Gambling Studies, 9, 63-84.

Yokel, R. A. y Wise, R. A. (1978) Amphetamine-type reinforcement by dopaminergic agonists in the rat. Psychopharmacology, 58, 289-296.

Zack, M. y Poulos, C. X. (2004). Amphetamine primes motivation to gamble and gambling-related semantic networks in problem gamblers. Neuropsychopharmacology. 29, 195-207.

Zack, M. y Poulos, C. X. (2009). Parallel roles for dopamine in pathological gambling and psychostimulant addiction. Current Drug Abuse Reviews, 2, 11-25. 